\title{
Distributed Sliding Mode Fault-Tolerant LFC for Multiarea Interconnected Power Systems under Sensor Fault
}

\author{
Xichao Zhou, ${ }^{1,2}$ Zhenlan Dou, ${ }^{3}$ Weiming Zhang $\mathbb{D},{ }^{4}$ Yiwei Zhang, ${ }^{5}$ Deyi Wang $\mathbb{D}^{1}$, \\ Chunyan Zhang, ${ }^{3}$ and Dezhi Xu ${ }^{4}{ }^{4}$ \\ ${ }^{1}$ School of Electrical Engineering, Xi'an University of Technology, Xi'an 710043, China \\ ${ }^{2}$ Science and Technology Research and Development Center, State Grid Integrated Energy Service Group Co., Ltd., \\ Beijing 100053, China \\ ${ }^{3}$ Technical Engineering Department, State Grid Shanghai Integrated Energy Service Co., Ltd., Shanghai 200023, China \\ ${ }^{4}$ School of Internet of Things Engineering, Jiangnan University, Wuxi 214122, China \\ ${ }^{5}$ State Grid Zhejiang Yuyao Power Supply Co., Ltd., Ningbo 315400, China
}

Correspondence should be addressed to Weiming Zhang; wmzhang21@163.com

Received 10 September 2021; Revised 13 December 2021; Accepted 12 January 2022; Published 10 February 2022

Academic Editor: Yue Song

Copyright (C) 2022 Xichao Zhou et al. This is an open access article distributed under the Creative Commons Attribution License, which permits unrestricted use, distribution, and reproduction in any medium, provided the original work is properly cited.

This paper focuses on the problem of load frequency control and sensor fault-tolerant control in the multiarea power grid. To solve these problems, a sliding mode control strategy based on an interval observer is designed. First, an interval observer is designed to obtain the boundary estimation information of the system state online, which is used to reconstruct the load disturbance online. Based on the reconstructed values, an integral sliding mode controller is designed to keep the system frequency stable when the load is disturbed. Then, sensor fault is diagnosed by interval residual. An augmented observer is designed to estimate sensor fault online by equivalent transformation, and the sensor fault is compensated by the fault estimation to reduce the influence of fault on system performance and ensure the reliable and stable operation of the power system. The simulation results show the superiority of the proposed control strategy.

\section{Introduction}

1.1. Literature Review. Because of the increasing size, interconnection, and complexity of power systems, today's power grids are composed of multiple highly interconnected areas, and various communication devices are used to protect, monitor, and control the power grid $[1,2]$. The areas are connected via tie lines to eliminate any mismatching between generating and demand. Frequency, as an important index of power quality, should be stable within a certain range, so the active power balance should be guaranteed in the operation of power system [3]. However, because of the continuous growth of the installed capacity of power systems, the continuous access of new energy and distributed energy storage system have brought new challenges to maintaining system frequency stable $[4,5]$. For example, the high permeability of renewable energy, such as wind power, can lead to power quality and stability problems in the power grid, which adversely affect the operation frequency of the grid $[6,7]$. Thus, frequency regulation is becoming more prominent in the reconstituted power systems [8]. Besides, the change of the load will cause the corresponding deviation of the system frequency and the power of the link line, resulting in the corresponding change of the power generated [9]. At the same time, network security $[10,11]$ and sensor fault $[12-14]$ cannot be ignored. When the sensor is faulty due to malicious attack signals, the fault will lead to a large frequency deviation. Even in the normal operation of communication network, sensors will inevitably be damaged or affected by interference signals, resulting in fault. The sensor faults can make a harmful effect on the power grid or even cause damage to the power grid during operating $[15,16]$. Therefore, it is particularly important to design an effective LFC strategy with fault diagnosis and FTC scheme to reliably control the frequency of power system within an acceptable range. 
1.2. Research Gap and Motivation. At present, the main method for improving the frequency stability of each area in power system is the load frequency control, which continuously adjusts the active output of the frequencymodulated generator set in the system to maintain the realtime active power balance between the generating side and the demand side [17]. However, with the increasing power demand and the increasing complexity of the system structure, the uncertainty of the system model is bound to increase, which reduces the accuracy and speed of load frequency control. In order to design a load frequency controller with good control performance, scholars at home and abroad have made great achievements in the physics-based dynamic model and data-driven methods, such as fuzzy logic control $[18,19]$, optimal control [20], model-free adaptive control [21], model predictive control $[22,23]$, robust control $[24,25]$, and other advanced control theories $[26,27]$. Among them, a distributed optimal load frequency control scheme that can realize frequency regulation and economic scheduling was proposed in [20]. Because of the characteristics of random fluctuations in load disturbances, an LFC strategy based on a disturbance observer is proposed in [27]. However, the complex structure of the disturbance observer limits the practical application of these methods. The interval observer can determine the bound values of the state by assuming that the uncertainty in the system is unknown but bounded [28-30]. This advantage has been applied to state estimation [29] and fault detection [31]. However, unknown input reconstruction based on the interval estimation is still a problem to be solved.

In addition, in recent decades, research on system faults has been focused on fault detection and FTC and lots of significant fruits have been achieved [32-36]. For a class of nonlinear Markovian jump systems, an observer-based fault-tolerant control scheme is proposed to make the closed-loop system randomly stable [32]. The distributed fault-tolerant control for interconnected systems is proposed to ensure the overall stability and constraints of the system when faults occur [33]. In order to solve the problem of sensor fault in nonlinear systems with uncertain parameters, a fault estimation technique based on an adaptive sliding mode observer was investigated in [37]. In addition to the large and complicated power network of a power system, the power system is operated, protected, and controlled through an intricate communication system [38]. Therefore, reducing the impact of sensor failures on the system is a serious challenge.

Based on the above research, we propose an interval disturbance reconstruction technique to rapidly and accurately decouple and reconstruct the load disturbances in each region, so that the sliding mode load frequency control strategy based on interval disturbance reconstruction can effectively and quickly respond to the load changes and keep the system frequency deviation within a small range. Aiming at the sensor fault problem, we design a controller based on fault compensation to reduce the influence of the fault on the system and improve the system performance.
1.3. Contribution and Paper Organization. In this paper, load disturbance and sensor fault are considered to stabilize the system frequency. Considering load fluctuation reconstruction, parameter uncertainty, sensor fault diagnosis, fault estimation, and fault transition, this paper proposes a fault-tolerant load frequency control strategy based on interval disturbance reconstruction. The main contributions are summarized as follows:

(1) Interval observers are designed in each area of the LFC model of the multiarea interconnected power system, and the boundary value of system state quantity in each area is estimated rapidly and stably by using the designed observer.

(2) Using the estimation values of the bound values of the system state combined with the disturbance reconstruction technology, the system uncertainty items including load disturbance and uncertain parameters are reconstructed online.

(3) The interval residual errors based on the interval observer can be used for online monitoring and diagnosis of system sensor faults.

(4) The system sensor fault is regarded as a system state. An augmented observer is designed to estimate the sensor fault online, and the fault estimate is used for fault compensation. The sliding mode fault-tolerant load frequency control strategy is proposed to maintain the reliability and stability of the system under the problems of load disturbance and sensor fault.

The remainder of this research is organized as follows. The LFC model in the multiarea interconnected power grid and some preliminaries are introduced in Section 2. The interval observer-based disturbance reconstruction and the new-type load frequency controller are presented in Section 3. Sensor fault diagnosis, fault estimation, and the corresponding FTC strategy are given in Section 4 . The simulation results are demonstrated in Section 5, and the conclusions are shown in Section 6.

\section{Problem Formulation and Preliminaries}

2.1. LFC System. The LFC problem for the multiarea power grid is considered in this paper. A three-area interconnected power grid is shown in Figure 1. Although the power grid has nonlinear and time varying characteristics, under stable operating conditions, the load variation range is small. It is possible to consider a linearized model near the steady-state operating point of system. A multiarea power grid usually consists of N LFC areas. For the sake of simplicity, use one equivalent generator to replace other generators in an area.

In each LFC subsystem, the frequency dynamic equation can be written as

$$
\Delta \dot{f}_{i}=-\frac{D_{i}}{H_{i}} \Delta f_{i}+\frac{1}{H_{i}} \Delta P_{m i}-\frac{1}{H_{i}} \Delta P_{t i e, i}-\frac{1}{H_{i}} \Delta P_{d i},
$$

where $\Delta f_{i}, \Delta P_{d i}, D_{i}$, and $H_{i}$ are the deviation of frequency, load disturbances, equivalent inertia constant, and equivalent damping coefficient; $\Delta P_{m i}$ and $\Delta P_{t i e, i}$ are generator 
output power and tie-line power, respectively, which can be described as

$$
\begin{gathered}
\Delta P_{m i}^{\cdot}=-\frac{1}{T_{t i}} \Delta P_{m i}+\frac{1}{T_{t i}} \Delta P_{g i}, \\
\Delta P_{t i e, i}^{\cdot}=2 \pi \sum_{j \neq i}^{N} T_{i j}\left(\Delta f_{i}-\Delta f_{j}\right),
\end{gathered}
$$

where $T_{t i}$ is the time constants of turbine, $T_{i j}$ is the synchronizing power coefficient in connection areas, $N$ means there are $N$ control areas, and $\Delta P_{g i}$ is governor valve position, which is determined by $\Delta f_{i}$ and power generation set points $\Delta P_{c i}$ and defined as

$$
\Delta \dot{P_{g i}}=-\frac{1}{R_{i} T_{g i}} \Delta f-\frac{1}{T_{g i}} \Delta P_{g i}-\frac{1}{T_{g i}} u_{i},
$$

where $R_{i}$ and $T_{g i}$ are the droop coefficient and time constants of governor and $u_{i}$ is a power command signal generated by the automatic generation control (AGC) system to achieve the balance between the generating side and the demand side.

Combining (1)-(4), the state-space equation of $i$-th area can be described as

$$
\begin{aligned}
& \dot{x}_{i}(t)=A_{i i} x_{i}(t)+B_{i} u_{i}(t)+\sum_{j \neq i}^{N} A_{i j} x_{j}(t)+F_{i} \Delta P_{d i}(t), \\
& y_{i}(t)=C_{i} x_{i}(t)+f_{s i}(t),
\end{aligned}
$$

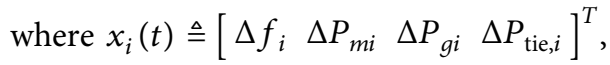

$$
\begin{aligned}
& A_{i i} \triangleq\left[\begin{array}{rrrr}
-\frac{D_{i}}{H_{i}} & \frac{1}{H_{i}} & 0 & -\frac{1}{H_{i}} \\
0 & -\frac{1}{T_{t i}} & \frac{1}{T_{t i}} & 0 \\
-\frac{1}{R_{i} T_{g i}} & 0 & -\frac{1}{T_{g i}} & 0 \\
2 \pi \sum_{j \neq i}^{N} T_{i j} & 0 & 0 & 0
\end{array}\right], \\
& A_{i j} \triangleq\left[\begin{array}{rrrr}
0 & 0 & 0 & 0 \\
0 & 0 & 0 & 0 \\
0 & 0 & 0 & 0 \\
-2 \pi T_{i j} & 0 & 0 & 0
\end{array}\right], \\
& C_{i} \triangleq\left[\begin{array}{llll}
1 & 0 & 0 & 0 \\
0 & 1 & 0 & 0 \\
0 & 0 & 1 & 0 \\
0 & 0 & 0 & 1
\end{array}\right],
\end{aligned}
$$

$$
\begin{aligned}
& B_{i} \triangleq\left[\begin{array}{llll}
0 & 0 & -\frac{1}{T_{g i}} & 0
\end{array}\right]^{T}, \\
& F_{i} \triangleq\left[\begin{array}{llll}
-\frac{1}{H_{i}} & 0 & 0 & 0
\end{array}\right]^{T} .
\end{aligned}
$$

Also, $\quad x_{i}(t) \in \mathbb{R}^{n}, \quad u_{i}(t) \in \mathbb{R}^{m}, \quad y_{i}(t) \in \mathbb{R}^{p}, \quad$ and $f_{s i}(t) \in \mathbb{R}^{p}$.

The actual power system is large and complex. Considering the system parameter uncertainties, the system model can be expressed as

$$
\begin{aligned}
\dot{x}_{i}(t)= & \left(A_{i i}+\Delta A_{i i}\right) x_{i}(t)+\left(B_{i}+\Delta B\right)_{i} u_{i}(t) \\
& +\sum_{j \neq i}^{N}\left(\left(A_{i j}+\Delta A_{i j}\right) x_{j}(t)+\left(F_{i}+\Delta F_{i}\right) \Delta P_{d i}(t),\right.
\end{aligned}
$$

where $\Delta A_{i i}, \Delta B_{i}, \Delta A_{i j}, \Delta F_{1 i}$, and $\Delta F_{2 i}$ denote the parameters of uncertainties.

Define

$$
\begin{aligned}
\mathrm{d}_{i}(t)= & \Delta A_{i i} x_{i}(t)+\Delta B_{i} u_{i}(t)+\sum_{j \neq i}^{N} \Delta A_{i j} x_{j}(t) \\
& +\left(F_{i}+\Delta F\right)_{i} \Delta P_{d i}(t) .
\end{aligned}
$$

Then, the model in (6) can be rewritten as

$$
\begin{aligned}
& \dot{x}_{i}(t)=A_{i i} x_{i}(t)+B_{i} u_{i}(t)+\sum_{j \neq i}^{N} A_{i j} x_{j}(t)+d_{i}(t), \\
& y_{i}(t)=C_{i} x_{i}(t)+f_{s i}(t) .
\end{aligned}
$$

Definition 1 (see [29]). A matrix is called Metzler matrix with nonnegative off-diagonal elements. A matrix is defined as a Hurwitz matrix if all eigenvalues have strictly negative real parts.

Lemma 1 (see [39]). For a system of the form $\dot{x}(t)=A x(t)+\omega(t)$, where matrix $A$ is Metzler, it has $x(t) \geq 0$ for all $t>0$ with $x(0)>0, \omega(t)>0$.

Lemma 2 (see [40]). For the transfer function of the form $\mathscr{G}(s)=\mathscr{C}(s I-\mathscr{A})^{-1} \mathscr{B}+\mathscr{D}$, we can get the following two equivalent conditions:

(1) $\left\|\mathscr{C}(s I-\mathscr{A})^{-1} \mathscr{B}+\mathscr{D}\right\|_{\infty}<\rho$ with $\mathscr{A}$ stable.

(2) There exists a positive matrix $P$ satisfying

$$
\left[\begin{array}{ccc}
P \mathscr{A}+\mathscr{A}^{T} P & P \mathscr{B} & \mathscr{C}^{T} \\
* & -\rho I & \mathscr{D}^{T} \\
* & * & -\rho I
\end{array}\right]<0 .
$$

Assumption 1. The system state vector $x_{i}(t)$ is available for measurement. 


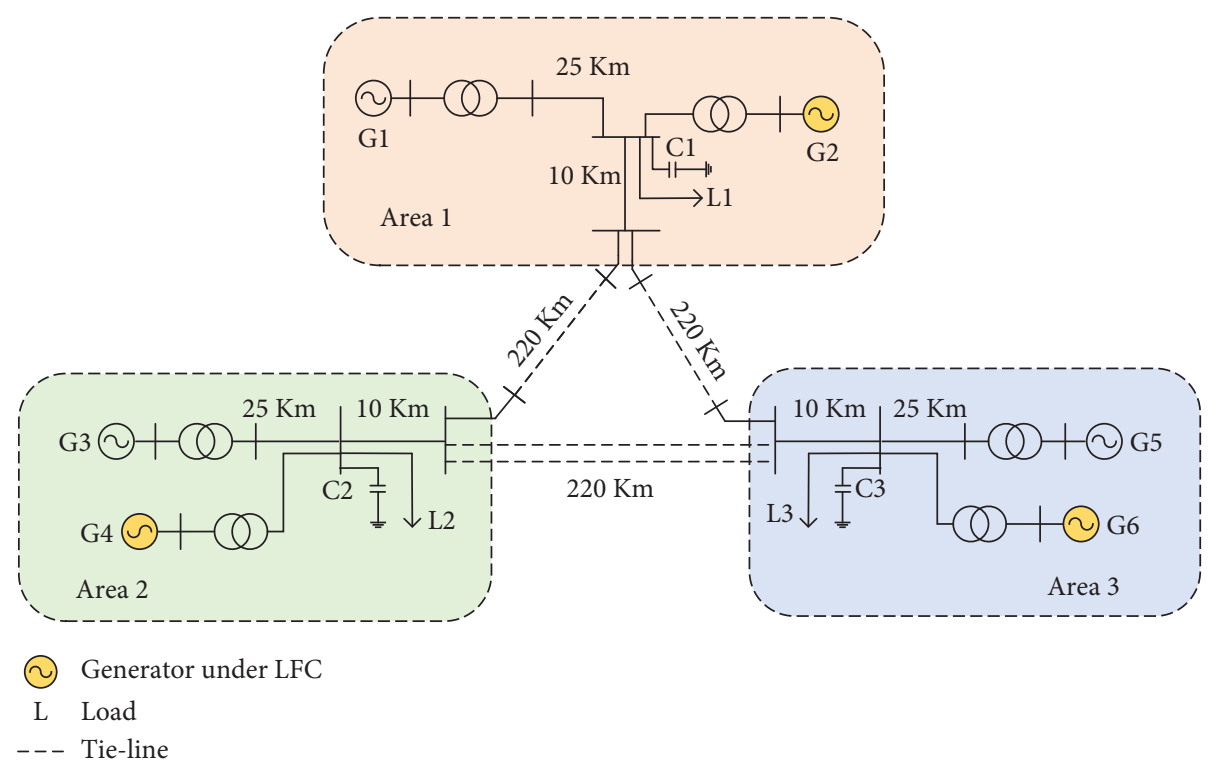

FIgURE 1: Three-area power system.

Assumption 2. There exist two known vectors $d_{i}^{+}>0$ and $d_{i}^{-}<0$ such that $d_{i}^{-} \leq d_{i}(t) \leq d_{i}^{+}$.

\section{Interval Observer-Based Disturbance Reconstruction and Sliding Mode LFC (DR- SMC-LFC)}

In this section, the load disturbance in the power system will be reconstructed with the designed interval observer. To make the perfect transient and steady-state performance under the load disturbance, an integral-type sliding mode controller will be developed in this section by using the reconstructed value.

3.1. Interval Observer Design. Design an interval observer of the following form:

$$
\begin{aligned}
\dot{\hat{x}}_{i}^{+}(t)= & A_{i i} y_{i}(t)+B_{i} u_{i}(t)+\sum_{j \neq i}^{N} A_{i j} y_{j}(t) \\
& +d_{i}^{+}-d_{i}^{-}+L_{i}\left(y_{i}(t)-C_{i} \hat{x}_{i}^{+}(t)\right), \\
\dot{\hat{x}}_{i}^{-}(t)= & A_{i i} y_{i}(t)+B_{i} u_{i}(t)+\sum_{j \neq i}^{N} A_{i j} y_{j}(t) \\
& +d_{i}^{-}-d_{i}^{+}+L_{i}\left(y_{i}(t)-C_{i} \hat{x}_{i}^{-}(t)\right),
\end{aligned}
$$

where $\widehat{x}_{i}^{+}(t)$ and $\widehat{x}_{i}^{-}(t)$ are the boundary estimations of $x_{i}(t)$, respectively, and $L_{i}$ is the gain of interval observer which needs to be defined in advance.

Theorem 1. Supposing that Assumptions 1 and 2 are held and a system without sensor faults, if the initial states of the proposed interval observer (10) meet the following inequalities,

$$
\widehat{x}_{i}^{-}(0) \leq x_{i}(0) \leq \hat{x}_{i}^{+}(0)
$$

then (10) is an interval observer for system (8) with $-L_{i}$ designed as Metzler and Hurwitz.

Proof. Denote estimation errors of the interval observer as $e_{1 i}(t)=\hat{x}_{i}^{+}(t)-x_{i}(t)$ and $e_{2 i}(t)=x_{i}(t)-\hat{x}_{i}^{-}(t)$. If the system runs normally without any sensor fault, from (8) and (10), the error dynamics system can be obtained as follows:

$$
\begin{aligned}
\dot{e}_{1 i}(t)= & A_{i i}\left(C_{i}-I\right) x_{i}(t)+\sum_{j \neq i}^{N} A_{i j}\left(C_{i}-I\right) x_{j}(t) \\
& +d_{i}^{+}-d_{i}^{-}-d_{i}(t)-L_{i} C_{i} e_{1 i}(t), \\
\dot{e}_{2 i}(t)= & A_{i i}\left(C_{i}-I\right) x_{i}(t)+\sum_{j \neq i}^{N} A_{i j}\left(C_{i}-I\right) x_{j}(t) \\
& -d_{i}^{-}+d_{i}^{+}+d_{i}(t)-L_{i} C_{i} e_{2 i}(t) .
\end{aligned}
$$

Recalling $C_{i}=I_{n}$,

$$
\begin{aligned}
& \dot{e}_{1}(t)=d_{i}^{+}-d_{i}^{-}-d_{i}(t)-L_{i} e_{1}(t), \\
& \dot{e}_{2}(t)=-d_{i}^{-}+d_{i}^{+}+d_{i}(t)-L_{i} e_{2}(t) .
\end{aligned}
$$

According to Assumption 2, it can be obtained that

$$
\begin{aligned}
d_{i}^{+}-d_{i}^{-}-d_{i}(t) & \geq 0, \\
-d_{i}^{-}+d_{i}^{+}+d_{i}(t) & \geq 0 .
\end{aligned}
$$

Choosing $-L_{i}$ as a Metzler and Hurwitz matrix, we can obtain that $\hat{x}_{i}^{-}(t) \leq x_{i}(t) \leq \hat{x}_{i}^{+}(t)$, for $t \geq 0$ from Lemma 1 , and the condition of Hurwitz ensures the system is stable when operating.

3.2. Disturbance Reconstruction. From Theorem 1, we can get $\hat{x}_{i}^{-}(t) \leq x_{i}(t) \leq \hat{x}_{i}^{+}(t)$, for all $t \geq 0$, so it is reasonable to obtain the following equation: 


$$
x_{i}(t)=\Phi_{i}(t) \hat{x}_{i}^{+}(t)+\left(I-\Phi_{i}(t)\right) \hat{x}_{i}^{-}(t),
$$

where $\quad \Phi_{i}(t)=\operatorname{diag}\left\{a_{1}(t), a_{2}(t), \ldots, a_{n}(t)\right\}$

and

$0 \leq a_{i}(t) \leq 1, i=1,2, \ldots, n$.

After obtaining the interval observer according to Theorem 1, the following theorem is proposed in combination with (15), which is used to reconstruct the load disturbance.

Theorem 2. For an LFC model (8) satisfying Assumptions 1 and 2 and an interval observer satisfying Theorem 1, the external load disturbance can be reconstructed by the following algebraic computation:

$$
\widehat{d}_{i}(t)=\widehat{M}_{1} \widehat{x}_{i}^{+}-\widehat{M}_{1} \widehat{x}_{i}^{-}+\widehat{M}_{2}\left(d_{i}^{+}-d_{i}^{-}\right),
$$

where $\widehat{M}_{1}$ and $\widehat{M}_{2}$ are defined as

$$
\begin{aligned}
& \widehat{M}_{1}=\widehat{\Phi}_{i}(t) \widehat{N}_{1}+\left(I-\widehat{\Phi}_{i}(t)\right) \widehat{N}_{2}+\dot{\Phi}_{i}(t)-A_{i i} \widehat{\Phi}_{i}(t), \\
& \widehat{M}_{2}=2 \widehat{\Phi}_{i}(t)-I,
\end{aligned}
$$

with $\quad \widehat{N}_{1}=A_{i i} \widehat{\Phi}_{i}(t)+L_{i} \widehat{\Phi}_{i}(t)-L_{i}$

$\widehat{N}_{2}=A_{i i} \widehat{\Phi}_{i}(t)+L_{i} \widehat{\Phi}_{i}(t)$.

Proof. According to (15) and (10), we can obtain

$$
\begin{aligned}
\dot{\hat{x}}_{i}^{+}(t)= & N_{1} \hat{x}_{i}^{+}+\left(A_{i i}-N_{1}\right) \hat{x}_{i}^{-}+B_{i} u_{i}(t)+d_{i}^{+}-d_{i}^{-} \\
& +\sum_{j \neq i}^{N} A_{i j}\left(\Phi_{j}(t) \hat{x}_{j}^{+}(t)+I-\Phi_{j}(t)\right) \hat{x}_{j}^{-}(t), \\
\dot{\hat{x}}_{i}^{-}(t)= & N_{2} \widehat{x}_{i}^{+}+\left(A_{i i}-N_{2}\right) \widehat{x}_{i}^{-}+B_{i} u_{i}(t)+d_{i}^{-}-d_{i}^{+} \\
& +\sum_{j \neq i}^{N} A_{i j}\left(\Phi_{j}(t) \hat{x}_{j}^{+}(t)+I-\Phi_{j}(t)\right) \hat{x}_{j}^{-}(t),
\end{aligned}
$$

where $\quad N_{1}=A_{i i} \Phi_{i}(t)+L_{i} \Phi_{i}(t)-L_{i}$ $N_{2}=A_{i i} \Phi_{i}(t)+L_{i} \Phi_{i}(t)$.

According to (15), the first derivative of $x_{i}(t)$ is

$$
\begin{aligned}
\dot{x}_{i}(t)= & \left(\Phi_{i}(t) N_{1}+\left(I-\Phi_{i}(t)\right) N_{2}+\dot{\Phi}_{i}(t)\right) \hat{x}_{i}^{+}+B_{i} u_{i}(t) \\
& +\left(A_{i i}-\Phi_{i}(t) N_{1}-\left(I-\Phi_{i}(t)\right) N_{2}-\dot{\Phi}_{i}(t)\right) \hat{x}_{i}^{-} \\
& +\sum_{j \neq i}^{N} A_{i j}\left(\Phi_{j}(t) \hat{x}_{j}^{+}(t)+\left(I-\Phi_{j}(t)\right) \hat{x}_{j}^{-}(t)\right) \\
& +\left(2 \Phi_{i}(t)-I\right)\left(d_{i}^{+}-d_{i}^{-}\right) .
\end{aligned}
$$

and

Substituting (8) and (15) into (19), we can obtain the reconstruction of $d_{i}(t)$ :

$$
d_{i}(t)=M_{1} \hat{x}_{i}^{+}-M_{1} \hat{x}_{i}^{-}+M_{2}\left(d_{i}^{+}-d_{i}^{-}\right),
$$

where $M_{1}=\Phi_{i}(t) N_{1}+\left(I-\Phi_{i}(t)\right)_{2}+\dot{\Phi}_{i}(t)-A_{i i} \Phi_{i}(t)$ and $M_{2}=2 \Phi_{i}(t)-I$.

Then, the external disturbance can be reconstructed by using (20). As for the computation of $\Phi_{i}(t)$, we can convert (15) to the following equality to calculate $\Phi_{i}(t)$ :

$$
\begin{aligned}
x_{i}(t)= & {\left[\begin{array}{cccc}
\hat{x}_{i 1}^{+} & & & \\
& \hat{x}_{i 2}^{+} & & \\
& & \ddots & \\
& & & \hat{x}_{i n}^{+}
\end{array}\right]\left[\begin{array}{c}
a_{1}(t) \\
a_{2}(t) \\
\vdots \\
a_{n}(t)
\end{array}\right] } \\
& +\left[\begin{array}{cccc}
\hat{x}_{i 1}^{-} & & & \\
& \hat{x}_{i 2}^{-} & & \\
& & \ddots & \\
& & & \hat{x}_{i n}^{-}
\end{array}\right]\left[\begin{array}{c}
1-a_{1}(t) \\
1-a_{2}(t) \\
\vdots \\
1-a_{n}(t)
\end{array}\right] .
\end{aligned}
$$

Define

$$
\begin{aligned}
Q_{1}(t) & =\operatorname{diag}\left\{\hat{x}_{i 1}^{+}, \hat{x}_{i 2}^{+}, \ldots, \hat{x}_{i n}^{+}\right\}, \\
Q_{2}(t) & =\operatorname{diag}\left\{\hat{x}_{i 1}^{-}, \hat{x}_{i 2}^{-}, \ldots, \hat{x}_{i n}^{-}\right\}, \\
a_{v}(t) & =\left[a_{1}(t), a_{2}(t), \ldots, a_{n}(t)\right]^{T} .
\end{aligned}
$$

Then, we can obtain

$$
\begin{aligned}
\left(Q_{1}(t)-Q_{2}(t)\right) a_{v}(t) & =x_{i}(t)-\hat{x}_{i}^{-}(t), \\
a_{v}(t) & =\left(Q_{1}(t)-Q_{2}(t)\right)^{-1}\left(x_{i}(t)-\widehat{x}_{i}^{-}(t)\right) .
\end{aligned}
$$

Consequently, the disturbance can be reconstructed by using (16).

3.3. Sliding Mode Load Frequency Controller Design. In order to maintain the system frequency stable when load disturbances occur, a new-type load frequency controller is designed and applied in this subsection which is used in the integral-type sliding mode technique. The integral-type switching surfaces can be designed as

$$
s_{i}(t)=G_{i} y_{i}(t)-\int_{0}^{t} G_{i}\left(A_{i i}+B_{i} K_{i}\right) y_{i}(\theta) d \theta,
$$

where $G_{i}$ and $K_{i}$ are constant matrices with appropriate dimension.

Traditional SMC utilizes the boundary value of load disturbance to design a control law. However, the uncertain term $d_{i}(t)$ always changes in actual engineering. The controller based on the boundary value $\xi_{i}$ may not preform very well, so we redesigned the controller by using the reconstructed value $\widehat{d}_{i}(t)$ and the adaptive law as follows: 


$$
\left\{\begin{array}{l}
u_{i}(t)=K_{i} y_{i}(t)-\left(\left(G_{i} B\right)_{i}\right)^{-1}\left[G_{i} \widehat{d}_{i}(t)+\kappa_{i} s_{i}(t)\right. \\
\left.\quad+G_{i} \sum_{j \neq i}^{N} A_{i j} y_{j}(t)+\widehat{\tau}_{i}\left\|G_{i}\right\| \operatorname{sat}\left(s_{i}(t)\right)\right] \\
\operatorname{sat}\left(s_{i}(t)\right)=\left\{\begin{array}{l}
\frac{s_{i}(t)}{\varepsilon_{i}},\left\|s_{i}(t)\right\| \leq \varepsilon_{i} \\
\operatorname{sign}\left(s_{i}(t)\right),\left\|s_{i}(t)\right\|>\varepsilon_{i}
\end{array}\right.
\end{array}\right.
$$

where $\widehat{d}_{i}(t)$ is the reconstruction of $d_{i}(t)$ from (16) and $\left\|d_{i}(t)-\hat{d}_{i}(t)\right\|<\tau_{i}$, in which $\tau_{i}$ is the positive constant, and we define the following adaptive law of $\widehat{\tau}_{i}$ :

$$
\dot{\hat{\tau}}_{i}=n_{i}\left\|G_{i}\right\|\left\|s_{i}(t)\right\| \text {. }
$$

Theorem 3. The closed-loop control system will remain asymptotically stable under the load frequency controller (25) based on load disturbance reconstruction with adaptive law (26).

Proof. Define a Lyapunov function as

$$
V_{i}(t) \triangleq 0.5 s_{i}^{2}(t)+0.5 \frac{1}{n_{i}} \widetilde{\tau}_{i}^{2}
$$

where $\tilde{\tau}_{i}=\tau_{i}-\widehat{\tau}_{i}$ and $\dot{\tilde{\tau}}_{i}=\dot{\tau}_{i}-\dot{\hat{\tau}}_{i}=-\dot{\hat{\tau}}_{i}$.

According to the switching surface (24), we can obtain

$$
\begin{aligned}
\dot{V}_{i}(t)= & s_{i}(t) \dot{s}_{i}(t)-\frac{1}{n_{i}} \tilde{\tau}_{i} \dot{\vec{\tau}}_{i} \\
= & s_{i}(t)\left[G_{i} C_{i} A_{i i} x_{i}(t)+G_{i} C_{i} B_{i} u_{i}(t)+G_{i} C_{i} d_{i}(t)\right. \\
& +G_{i} C_{i} \sum_{j \neq i}^{N} A_{i j} x_{j}(t)-G_{i} A_{i i} C_{i} x_{i}(t) \\
& \left.-G_{i} B_{i} K_{i} C_{i} x_{i}(t)\right]-\frac{1}{n_{i}} \tilde{\tau}_{i} \dot{\hat{\tau}}_{i} .
\end{aligned}
$$

Recalling $C_{i}=I_{n}$,

$$
\begin{aligned}
\dot{V}_{i}(t)= & s_{i}(t)\left[G_{i} B_{i} u_{i}(t)+G_{i} \sum_{j \neq i}^{N} A_{i j} x_{j}(t)+G_{i} d_{i}(t)\right. \\
& \left.-G_{i} B_{i} K_{i} x_{i}(t)\right]-\frac{1}{n_{i}} \tilde{\tau}_{i} \dot{\vec{\tau}}_{i} \\
= & s_{i}(t)\left[G_{i}\left(d_{i}(t)-\widehat{d}_{i}(t)\right)-\kappa_{i} s_{i}(t)\right. \\
& \left.-\widehat{\tau}_{i}\left\|G_{i}\right\| \operatorname{sat}\left(s_{i}(t)\right)\right]-\frac{1}{n_{i}} \widetilde{\tau}_{i} \dot{\vec{\tau}}_{i} \\
\leq & \left\|G_{i}\right\|\left\|s_{i}(t)\right\| \tau_{i}-\kappa_{i}\left\|s_{i}(t)\right\|^{2}-\left\|G_{i}\right\|\left\|s_{i}(t)\right\| \widehat{\tau}_{i} \\
& -\frac{1}{n_{i}} \tilde{\tau}_{i} \dot{\vec{\tau}}_{i} \\
= & \left\|G_{i}\right\|\left\|s_{i}(t)\right\|\left(\tau_{i}-\widehat{\tau}_{i}-\tilde{\tau}_{i}\right)-\kappa_{i}\left\|s_{i}(t)\right\|^{2} \\
= & -\kappa_{i}\left\|s_{i}(t)\right\|^{2}<0 .
\end{aligned}
$$

Consequently, it can be concluded that the system state will eventually remain stable under the proposed controller, which implies the new-type LFC controller can ensure the stability of each control area in power system.

\section{Sensors Fault Diagnosis and Estimation and FTC Strategy}

The closed-loop control of the system requires precise measurements. However, any fault that may affect the sensor 
will inevitably lead to performance degradation and cause system damage. Therefore, to ensure the stability and reliability when the system is operating, it is necessary to perform fault detection and fault tolerance control when faults occur. In this section, the fault diagnosis based on interval residual errors, sensor fault estimation based on the augmented observer, and FTC strategy are defined, and the whole control strategy is redesigned as shown in Figure 2.

4.1. Fault Diagnosis. According to the interval observer designed in Section 3, the following interval residual errors are designed for sensor fault diagnosis:

$$
\begin{aligned}
& r_{i}^{+}(t)=y_{i}(t)-\widehat{y}_{i}^{-}(t), \\
& r_{i}^{-}(t)=y_{i}(t)-\hat{y}_{i}^{+}(t),
\end{aligned}
$$

where

$$
\begin{aligned}
& \hat{y}_{i}^{+}(t)=C_{i}^{+} \hat{x}_{i}^{+}(t)-C_{i}^{-} \hat{x}_{i}^{-}(t), \\
& \hat{y}_{i}^{-}(t)=C_{i}^{+} \hat{x}_{i}^{-}(t)-C_{i}^{-} \hat{x}_{i}^{+}(t) .
\end{aligned}
$$

According to Theorem 1 , if $-L_{i}$ is designed as a Metzler matrix, then under the initial condition $\hat{x}_{i}^{-}(0) \leq x_{i}(0) \leq \hat{x}_{i}^{+}(0)$ and without sensor faults, we can get

$$
\begin{aligned}
\hat{x}_{i}^{-}(t) & \leq x_{i}(t) \leq \hat{x}_{i}^{+}(t), \\
\hat{y}_{i}^{-}(t) & \leq y_{i}(t) \leq \hat{y}_{i}^{+}(t), \\
0 & \in\left[r_{i s}^{-}(t), r_{i s}^{+}(t)\right], s=1,2 \ldots, 4 .
\end{aligned}
$$

In case of faults, even if $-L_{i}$ is designed as Metzler and Hurwitz, the nonnegativity of the error dynamics cannot be guaranteed. Therefore, $0 \in\left[r_{i s}^{-}(t), r_{i s}^{+}(t)\right]$ will not be guaranteed at this time, which can be used for detecting faults.

4.2. Fault Estimation. The following augmented system (33) is designed which is equivalent to the original dynamic system (8). This equivalent transformation treats the sensor failure $f_{s i}(t)$ in the original system (8) as a state one, so that an effective state observer strategy can be designed, while estimating the states and sensor faults.

$$
\left\{\begin{array}{l}
\dot{\bar{x}}_{i}(t)=\bar{A}_{i i} \bar{x}_{i}(t)+\bar{B}_{i} u_{i}(t)+w_{i}(t)+\sum_{j \neq i}^{N} \bar{A}_{i j} \bar{x}_{j}(t) \\
y_{i}(t)=\bar{C}_{i} \bar{x}_{i}(t)
\end{array}\right.
$$

where

$$
\begin{aligned}
& \bar{x}_{i} \triangleq\left[\begin{array}{c}
x_{i} \\
f_{s i}
\end{array}\right], \bar{A}_{i i} \triangleq\left[\begin{array}{cc}
A_{i i} & 0 \\
0 & 0 \\
p \times p
\end{array}\right], \bar{B}_{i} \triangleq\left[\begin{array}{c}
B_{i} \\
0_{p \times m}
\end{array}\right] \\
& \bar{C}_{i} \triangleq\left[\begin{array}{ll}
C_{i} & I_{p}
\end{array}\right], \bar{A}_{i j} \triangleq\left[\begin{array}{cc}
A_{i j} & 0 \\
0 & 0
\end{array}\right], w_{i}=\left[\begin{array}{c}
d_{i} \\
\dot{f}_{s i}
\end{array}\right] .
\end{aligned}
$$

For the augmented system (33), a state observer for $i$ th subsystem is constructed as follows:

$$
\left\{\begin{array}{l}
\dot{\overline{\bar{x}}}_{i}(t)=\bar{A}_{i i} \widehat{\bar{x}}_{i}(t)+\bar{B}_{i} u_{i}(t)+L_{p i}\left(y_{i}(t)-\widehat{y}_{i}(t)\right) \\
+\sum_{j \neq i}^{N} \bar{A}_{i j} \hat{\bar{x}}_{j}, \\
\hat{y}_{i}(t)=\bar{C}_{i} \hat{\bar{x}}_{i}(t),
\end{array}\right.
$$

where $\hat{\bar{x}}_{i}(t)$ is the estimation of $\bar{x}_{i}(t)$ by the augmented observer and $L_{p i}$ is a constant matrix with appropriate dimension.

Define the state estimate error and sensor fault estimate error as $e_{x}(t)=\widehat{x}_{i}(t)-x_{i}(t), e_{f s i}(t)=\widehat{f}_{s i}(t)-f_{s i}(t)$, and $\bar{e}_{i}(t)=\left[\begin{array}{ll}e_{x}^{T}(t) & e_{f s i}^{T}(t)\end{array}\right]^{T}$. From (33) and (35), the error dynamic system is obtained as

$$
\left\{\begin{array}{l}
\dot{\bar{e}}_{i}(t)=\left(\bar{A}_{i}-L_{p i} \bar{C}_{i}\right) \bar{e}_{i}(t)+\sum_{j \neq i}^{N} \bar{A}_{i j} \bar{e}_{j}-w_{i}(t) \\
e_{f s i}(t)=\bar{I}_{p}^{T} \bar{e}_{i}(t) \quad i=1,2, \ldots, N
\end{array},\right.
$$

where $\bar{I}_{p}=\left[\begin{array}{ll}0 & I_{p}\end{array}\right]^{T}$.

Theorem 4. Given a $H_{\infty}$ performance level $\rho$, if there exist matrix $W_{i} \in \mathbb{R}^{(n+p) N \times p N}>0$ and symmetric matrices $P_{i} \in \mathbb{R}^{(n+p) N \times(n+p) N}>0$ such that condition (37) is satisfied,

$$
\left[\begin{array}{ccc}
\Gamma_{1} & -P & I_{N} \otimes \bar{I}_{p} \\
* & -\rho I_{(n+p) N} & 0 \\
* & * & -\rho I_{p N}
\end{array}\right]<0
$$

where

$$
\begin{aligned}
\Gamma_{1} & =P \bar{A}-W \bar{C}+(P \bar{A}-W \bar{C})^{T}, \\
P & =\operatorname{diag}\left\{P_{1}, P_{2}, \ldots, P_{N}\right\}, \\
W & =\operatorname{diag}\left\{W_{1}, W_{2}, \ldots, W_{N}\right\}, \\
\bar{C} & =\operatorname{diag}\left\{\bar{C}_{1}, \bar{C}_{2}, \ldots, \bar{C}_{N}\right\}, \\
\bar{A} & =\left[\begin{array}{cccc}
\bar{A}_{11} & \bar{A}_{12} & \ldots & \bar{A}_{1 N} \\
\bar{A}_{21} & \bar{A}_{21} & \ldots & \bar{A}_{2 N} \\
\vdots & \vdots & \ddots & \vdots \\
\bar{A}_{N 1} & \bar{A}_{N 2} & \cdots & \bar{A}_{N N}
\end{array}\right],
\end{aligned}
$$

then the error dynamics of the whole interconnected power system satisfies the $H_{\infty}$ performance. Also, we can calculate the gain matrices $L_{p}$ by $L_{p i}=P_{i}^{-1} W_{i}$.

Proof. Define augmented vectors and matrices as follows:

$$
\begin{aligned}
L_{p} & =\operatorname{diag}\left\{L_{p 1}, L_{p 2}, \ldots, L_{p N}\right\}, \\
\bar{e}(t) & =\left[\begin{array}{llll}
\bar{e}_{1}^{T}(t) & \bar{e}_{2}^{T}(t) & \cdots & \bar{e}_{N}^{T}(t)
\end{array}\right]^{T}, \\
e_{f s}(t) & =\left[\begin{array}{llll}
e_{f s 1}^{T}(t) & e_{f s 2}^{T}(t) & \cdots & e_{f s N}^{T}(t)
\end{array}\right]^{T}, \\
w(t) & =\left[\begin{array}{llll}
w_{1}^{T}(t) & w_{2}^{T}(t) & \cdots & w_{N}^{T}(t)
\end{array}\right]^{T} .
\end{aligned}
$$
follows:

So, we can get the global error dynamics of power grid as 


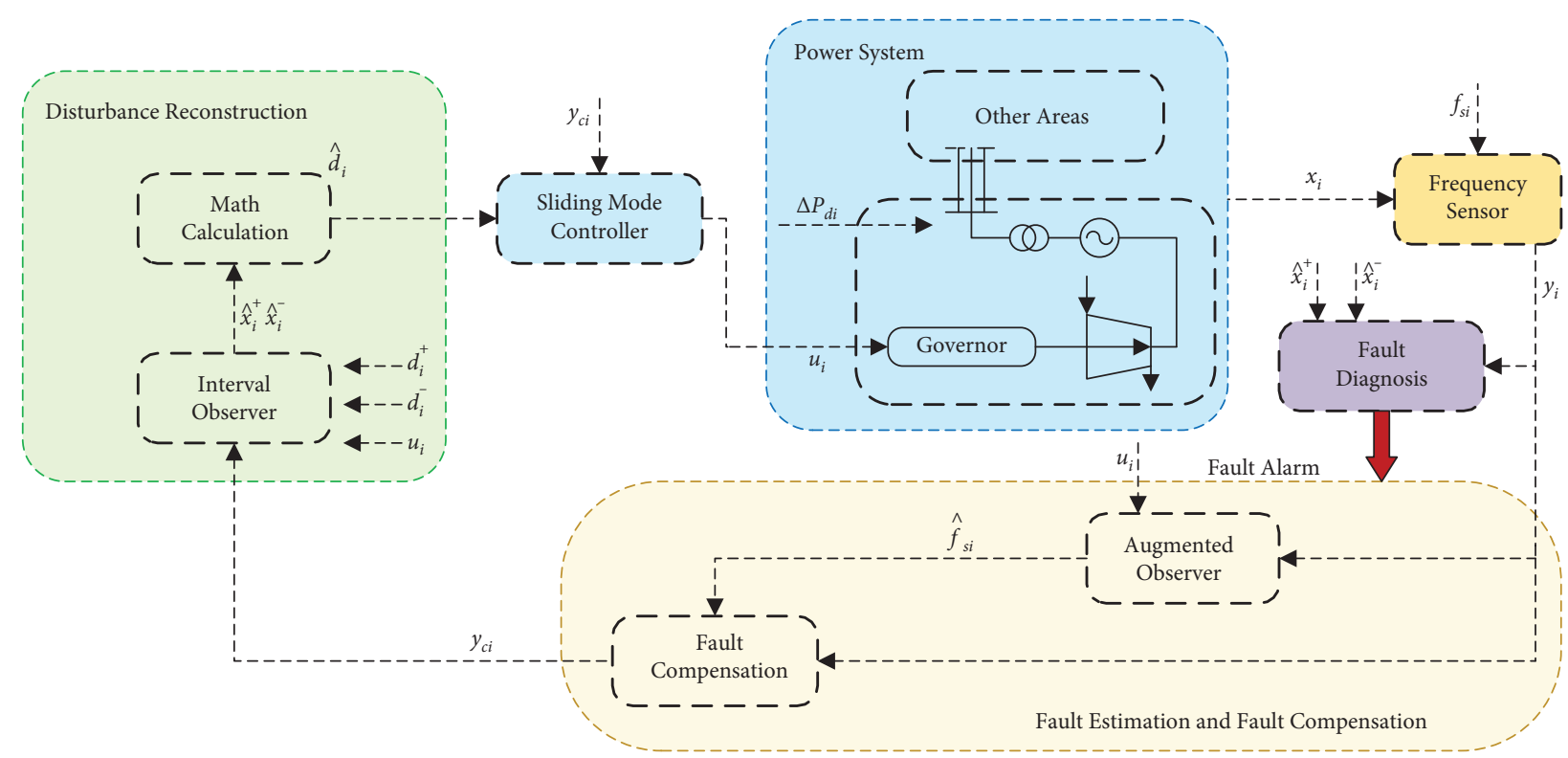

FIGURE 2: The structure of the proposed control approach.

$$
\left\{\begin{array}{l}
\dot{\bar{e}}(t)=\left(\bar{A}-L_{p} \bar{C}\right) \bar{e}(t)-w(t) \\
e_{f_{s}}(t)=I_{N} \otimes \bar{I}_{p}^{T} \bar{e}(t)
\end{array}\right.
$$

From the global view, $W=P L_{p}$, so (37) can be rewritten as

$$
\left[\begin{array}{ccc}
\Gamma_{2} & -P & I_{N} \otimes \bar{I}_{p} \\
* & -\rho I_{(n+p) N} & 0 \\
* & * & -\rho I_{p N}
\end{array}\right]<0
$$

where

$$
\Gamma_{2}=P \bar{A}-P L_{p} \bar{C}+\left(P \bar{A}-P L_{p} \bar{C}\right)^{T} .
$$

According to Lemma 2, if (37) is satisfied, the global error dynamics system (40) will satisfy the $H_{\infty}$ performance $\left\|-\left(I_{N} \otimes \bar{I}_{p}^{T}\right)\left(s I-\left(\bar{A}-L_{p} \bar{C}\right)\right)^{-1}\right\|_{\infty}<\rho$.

Remark 1 . The $H_{\infty}$ performance in Theorem 4 can inhibit the influence of $w(t)$ on sensor failure estimation error $e_{f s}(t)$. The condition in (37) is in the form of LMI which can make the design of the gain matrix $L_{p i}$ more convenient.

4.3. Fault Compensation. According to the augmented observer designed in the previous section, the online sensor failure estimations can be obtained by

$$
\widehat{f}_{s i}(t)=\bar{I}_{p}^{T} \widehat{\bar{x}}_{i}(t)
$$

To keep the system stable when operating, the signals of system output are compensated accordingly by using the sensor fault estimation information, when faults occur.

$$
\begin{aligned}
y_{c i}(t) & =y_{i}(t)-\widehat{f}_{s i}(t) \\
& =C_{i} x_{i}(t)+f_{s i}(t)-\widehat{f}_{s i}(t) \\
& =C_{i} x_{i}(t)+e_{f s i}(t),
\end{aligned}
$$

where $y_{c i}(t)$ is the compensated output signal.

Remark 2. The $H_{\infty}$ performance used in Theorem 4 can inhibit the influence of $w(t)$ on sensor failure estimation error $e_{f s}(t)$. We can obtain accurate sensor fault estimations as long as we choose appropriate $H_{\infty}$ performance level $\gamma$. Therefore, the fault compensation part can use the sensor fault estimations, which greatly eliminate the interference of the sensor faults on the system performance and guarantee the reliability and stability when the system is running.

4.4. Fault-Tolerant Control. Once the sensor faults occur, the compensation signal described in (44) is used to replace the output signal of the system measurement. Therefore, the 
DR-SMC-LFC controller proposed in this paper can be redefined as

$$
\begin{gathered}
s_{i}(t)=G_{i} y_{c i}(t)-\int_{0}^{t} G_{i}\left(A_{i i}+B_{i} K_{i}\right) y_{c i}(\theta) \mathrm{d} \theta, \\
\left.+G_{i} \sum_{j \neq i}^{N} A_{i j} y_{c j}(t)+\widehat{\tau}_{i}\left\|G_{i}\right\| \operatorname{sat}\left(s_{i}(t)\right)\right] \\
\operatorname{sat}\left(s_{i}(t)\right)=\left\{\begin{array}{l}
\frac{s_{i}(t)}{\varepsilon_{i}},\left\|s_{i}(t)\right\| \leq \varepsilon_{i} \\
\operatorname{sign}\left(s_{i}(t)\right),\left\|s_{i}(t)\right\|>\varepsilon_{i}
\end{array}\right.
\end{gathered}
$$

In this way, it can not only keep the system frequency stable when the load changes in the power system but also timely compensate the measurement signal when the faults occur and realize FTC.

\section{Simulation Results}

To investigate the performance of the proposed method in a multiarea LFC, a three-area interconnected power grid model was built on the MATLAB/Simulink environment. Two cases with or without sensor faults under load disturbance are simulated, and the simulation results are demonstrated in Figures 3-5. The system model parameters are standardized with $100 \mathrm{MW}$ as the reference value, and the established system parameters [15] are shown in Table 1. The purpose of LFC is to maintain the frequency within the nominal range and minimize the connection power between different control areas. Therefore, $\Delta f_{i}(t)$ and $\Delta P_{t i e, i}$ can be used to compare the quality of different control strategies.

5.1. Case 1. In this case, fully considering the interconnectivity of the multiarea power grid, different load disturbances are applied in the three areas, respectively.

In order to realize the estimation of the bound values of the state in each control area by the designed interval observer, take area 1 as an example and design the parameters and initial values of the interval observer (10) as follows:

$$
\begin{aligned}
\widehat{x}_{1}^{+}(0) & =[0.005 ; 0.005 ; 0.005 ; 0.005], \widehat{x}_{1}^{-}(0)=-\widehat{x}_{1}^{+}(0), \\
d_{1}^{+}(0) & =[0.01 ; 0.01 ; 0.01 ; 0.01], d_{1}^{-}(0)=-d_{1}^{+}(0), \\
L_{1}= & {\left[\begin{array}{rrrr}
10 & -2 & -2 & -2 \\
-2 & 10 & -2 & -2 \\
-2 & -2 & 10 & -2 \\
-2 & -2 & -2 & 10
\end{array}\right] . }
\end{aligned}
$$

Taking the deviation of system frequency and tie-line power as an example, the real-time boundary estimations' effect of frequency deviation is shown in Figure 3(a) and the real-time boundary estimations' effect of tie-line power deviation is shown in Figure 3(b). From the simulation results, it can be seen that the estimations of the bound values of the state can respond rapidly from its initial state, quickly follow the change of the state in each control area, and ensure the state condition of $\hat{x}_{i}^{-}(t) \leq x_{i}(t) \leq \hat{x}_{i}^{+}(t)$.

By using the real-time boundary estimations obtained by using the interval observer combined with the interval disturbance reconstruction technology proposed in this paper, the load disturbances in the three areas are reconstructed in real time. Figure 3(c) shows the reconstruction effect of interval disturbances which is compared with actual load disturbances. The advantage of interval disturbance reconstruction is that it does not need to obtain the precise boundary value of disturbance. The key of disturbance reconstruction is to ensure $d_{i}^{-} \leq d_{i}(t) \leq d_{i}^{+}$, which leaves some leeway for the designer to select the appropriate disturbance boundary. The simulation results show that although the three areas are interconnected, the interval disturbance reconstruction technology can still quickly and accurately decouple and reconstruct the load disturbances in each area, which provides an important basis for the design of the subsequent sliding mode load frequency controller.

The real-time disturbance reconstruction value obtained through interval disturbance reconstruction is used to design the new load frequency controller. To achieve control performance, take area 1 as an example; the parameters of controller (25) are designed as follows:

$$
K_{1}=[6,1,0,3], G_{1}=B_{1}^{\dagger}, \kappa_{1}=0.02, n_{1}=0.03 \text {. }
$$

To further study the effectiveness of the designed interval disturbance reconstruction based sliding mode load frequency control strategy, the control effect is compared with the PI-type load frequency controller (PI-LFC) and the traditional sliding mode controller (SMC-LFC). The design parameters of the PI load frequency controller are obtained through repeated experiments, and the traditional sliding mode controller is selected as follows with disturbance boundary value $\xi_{i}=0.01$ : 

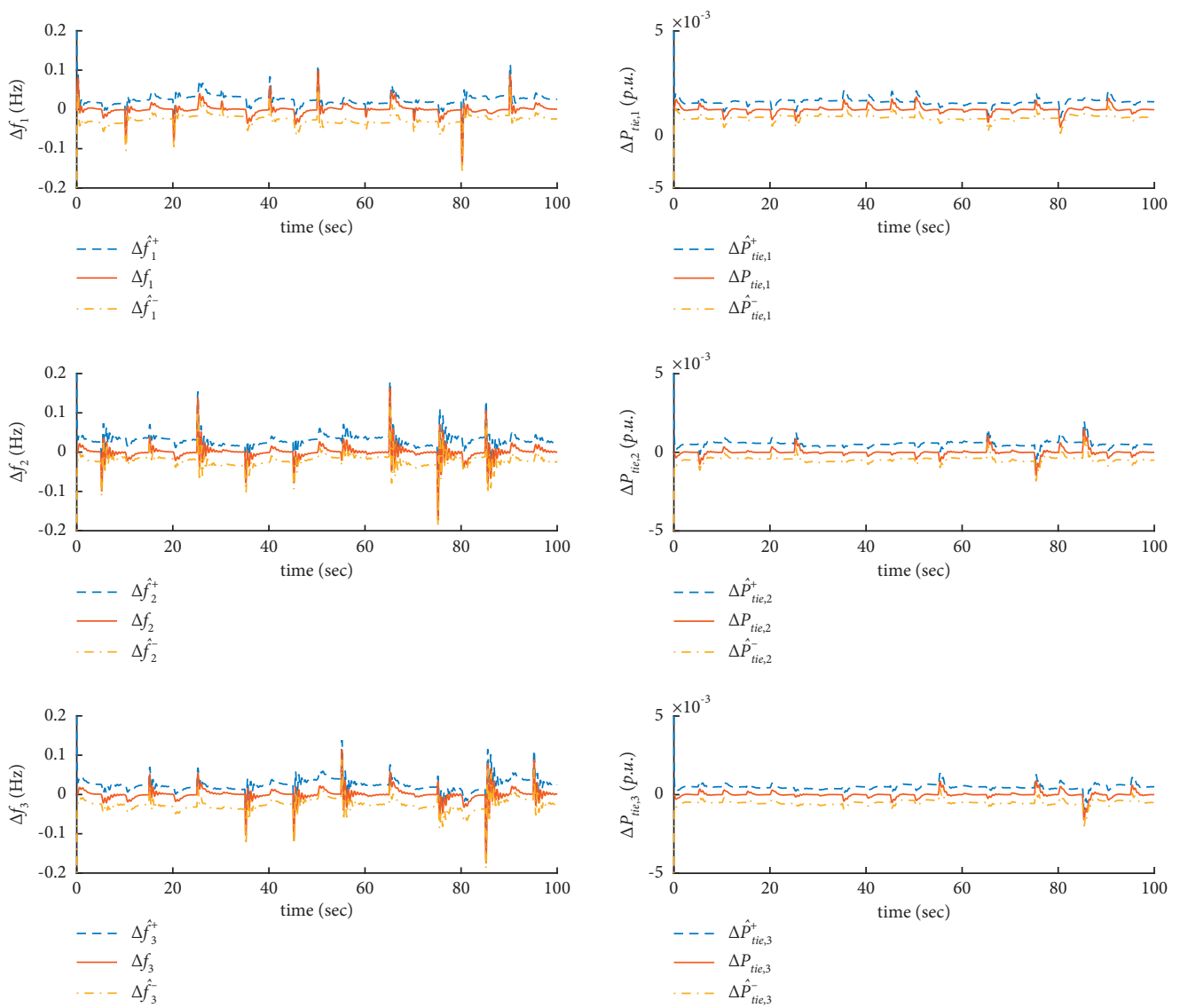

(a)

(b)
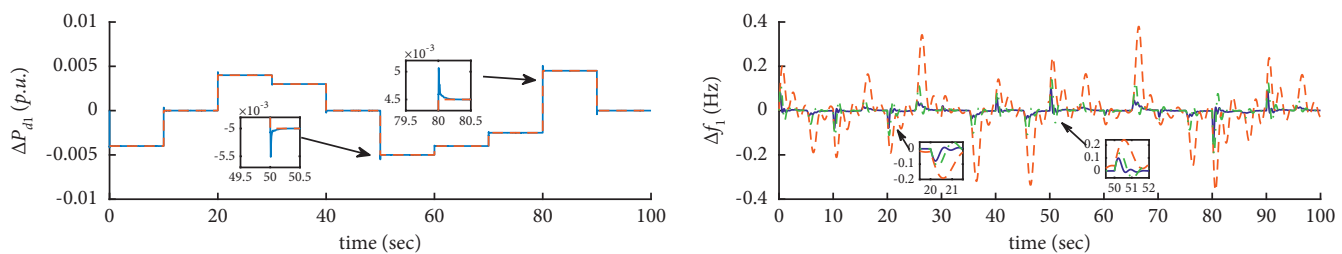
$\longrightarrow \Delta \hat{P}_{d 1}$

— DR-SMC-LFC

- - PI-LFC

-. SMC-LFC
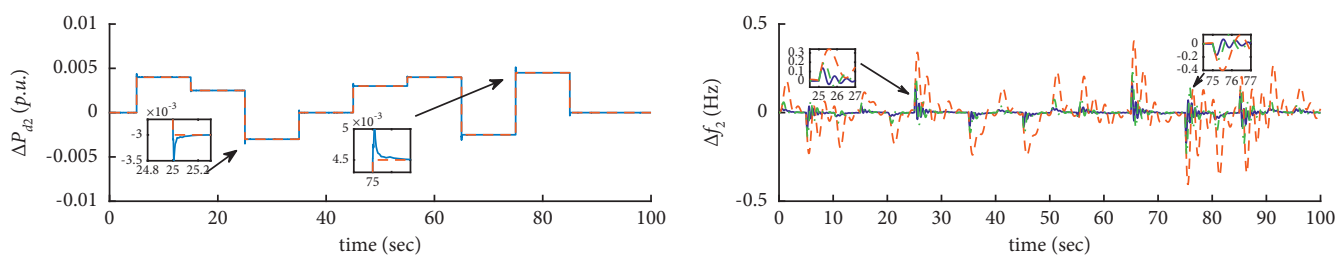

$-\Delta \hat{P}_{d 2}$
$---\Delta P_{d 2}$

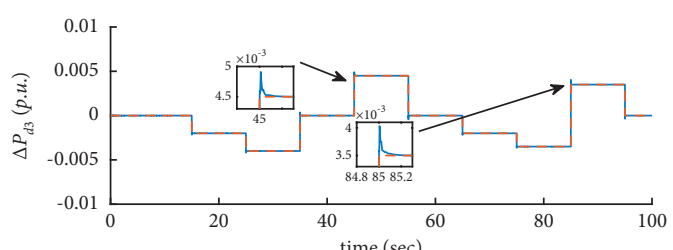

$-\Delta \hat{P}_{d 3}$
$---\Delta P_{d 3}$

- DR-SMC-LFC

- - - PI-LFC

-. - SMC-LFC

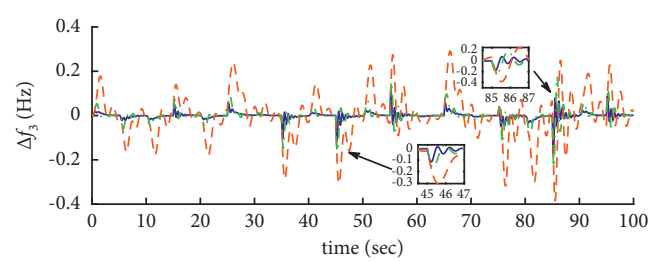

— DR-SMC-LFC

- - PI-LFC

-. - SMC-LFC

(c)

(d)

FIgURE 3: Simulation performance. (a) The interval estimation for $\Delta f_{i}(t)$ of each power grid. (b) The interval estimation for $\Delta P_{\text {tie, } i}(t)$ of each power grid. (c) The reconstruction of the load disturbance $\Delta P_{d i}(t)$ of each power grid. (d) $\Delta f_{i}(t)$ of each power grid under load disturbance. 

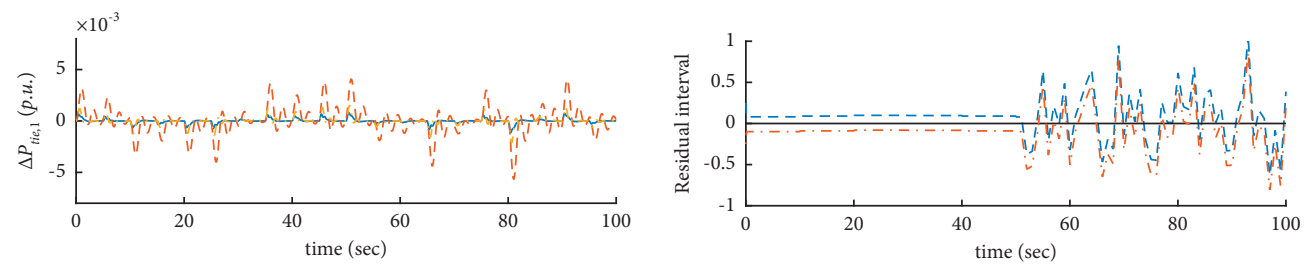

- DR-SMC-LFC
$\ldots-\ldots$ PI-LFC

$---r^{+}\left[\Delta f_{1}\right]$

SMC-LFC

$\begin{array}{ll}- & 0 \\ -- & r^{-}\left[\Delta f_{1}\right]\end{array}$
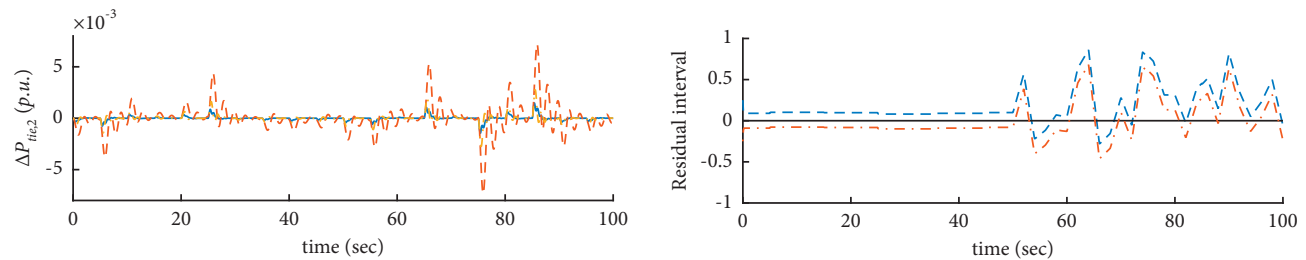

- DR-SMC-LFC

- - - PI-LFC

$---r^{+}\left[\Delta f_{2}\right]$

$\left.\begin{array}{ll}- & 0 \\ - & r\end{array}\right]$
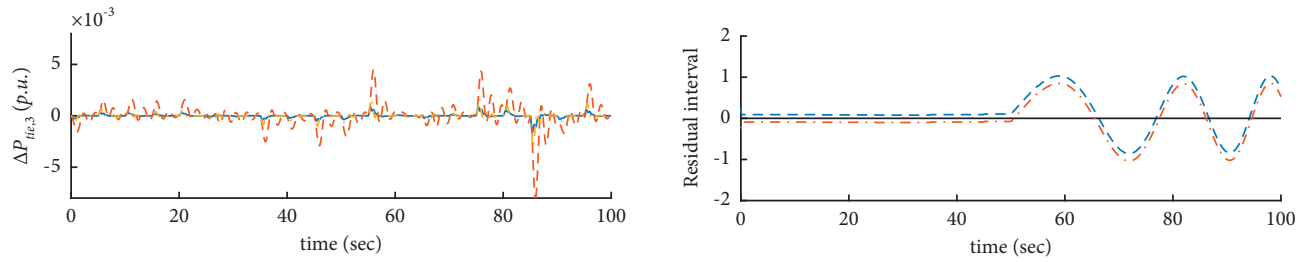

- DR-SMC-LFC

$---r^{+}\left[\Delta f_{3}\right]$

- - PI-LFC

-. $r^{-}\left[\Delta f_{3}\right]$

(a)

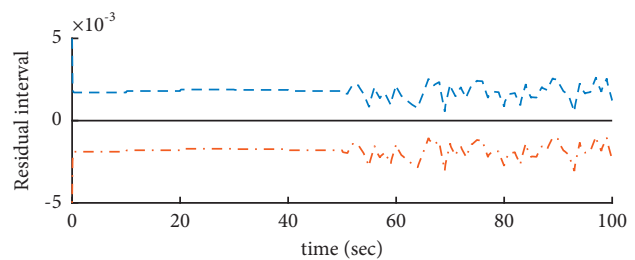

- - $r^{+}\left[\Delta P_{t i e, 1}\right]$

-.. $r^{-}\left[\Delta P_{t i e s,}\right.$

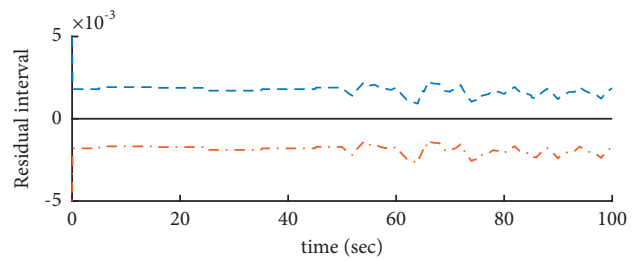

$\begin{array}{ll}---r^{+}\left[\Delta P_{t i e, 2}\right] \\ - & 0\end{array}$

$\cdots r^{-}\left[\Delta P_{t i e, 2}\right]$

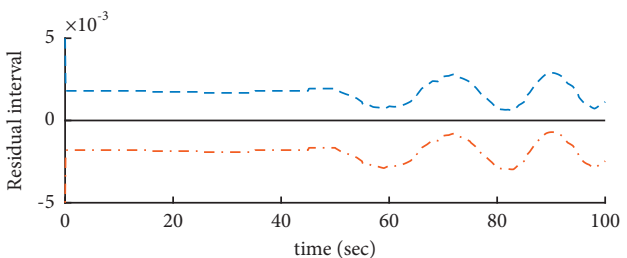

$\begin{array}{ll}- & r^{+}\left[\Delta P_{t i e, 3}\right] \\ - & 0\end{array}$

... $r^{-}\left[\Delta P_{t i e, 3}\right]$

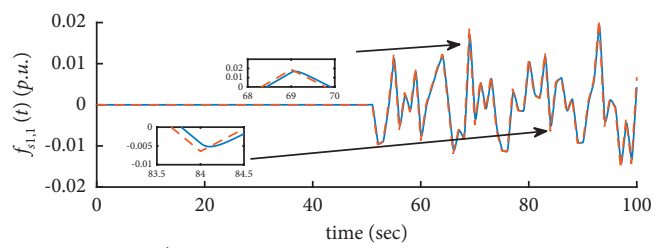

$$
\begin{aligned}
& -\hat{f}_{s 1,1}(t) \\
& --\cdot-f_{s 1,1}(t)
\end{aligned}
$$

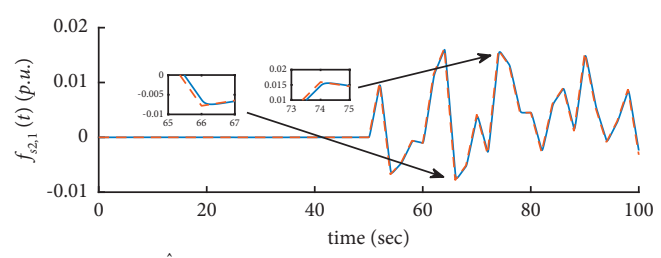

$$
\begin{aligned}
& --\hat{f}_{s 2,1}(t) \\
& -\cdot-f_{s 2,1}(t)
\end{aligned}
$$

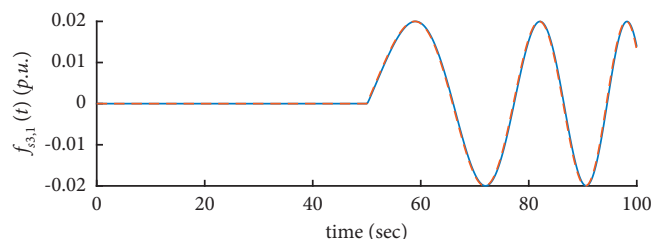

$$
\begin{aligned}
& -\hat{f}_{s 3,1}(t) \\
& -\cdots
\end{aligned}
$$

FIGURE 4: Simulation performance. (a) $\Delta P_{t i e, i}(t)$ of each power grid under load disturbance. (b) Interval residual errors of $\Delta f_{i}(t)$ of each power grid. (c) Interval residual errors of $\Delta P_{t i e, i}(t)$ of each power grid. (d) The estimation of $f_{s i}(t)$ of each power grid. 

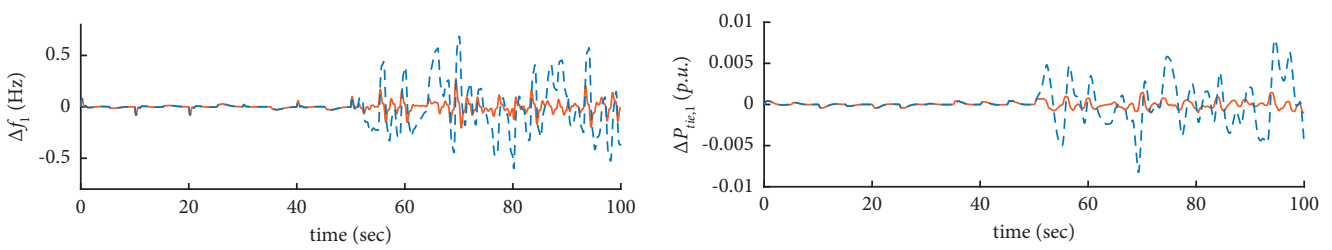

$\begin{array}{ll}\text { __ } & \text { with fault compensation } \\ -\_ & \text {without fault compensation }\end{array}$

— with fault compensation

- - - without fault compensation
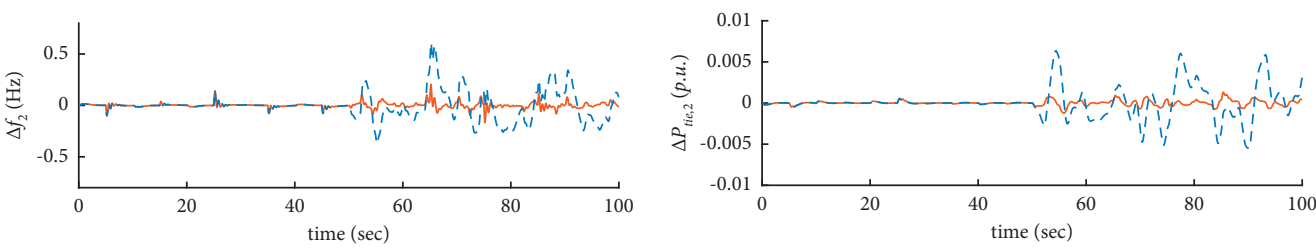

_ with fault compensation

- - - without fault compensation

— with fault compensation

- _ - without fault compensation
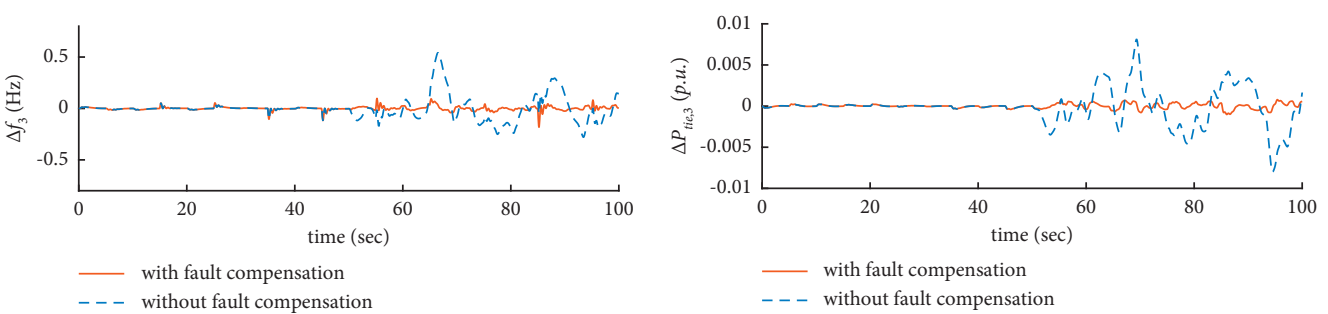

(a)

(b)

FIGURE 5: Simulation performance. (a) $\Delta f_{i}(t)$ and (b) $\Delta P_{t i e, i}(t)$ of each power grid under load disturbance and sensor fault.

TABLE 1: The parameters of the three-area power grid.

\begin{tabular}{lccc}
\hline & Area 1 & Area 2 & Area 3 \\
\hline$D_{i}$ (p.u./Hz) & 0.025 & 0.025 & 0.025 \\
$H_{i}$ (p.u. s) & 0.3708 & 0.2708 & 0.2708 \\
$T_{t i}$ (s) & 0.19 & 0.23 & 0.23 \\
$T_{g i}$ (s) & 0.05 & 0.05 & 0.05 \\
$R_{i}$ (Hz/p.u.) & 2.15 & 2.45 & 2.45 \\
& $T_{12}=0.13, T_{13}=0.12, T_{23}=0.10$ (p.u./Hz) & \\
\hline
\end{tabular}

$$
u_{i}(t)=K_{i} x_{i}(t)-\left(G_{i} B_{i}\right)^{-1}\left[G_{i} \xi_{i}+\kappa_{i} s_{i}(t)+G_{i} \sum_{j \neq i}^{N} A_{i j} x_{j}(t)+m_{i} \operatorname{sign}\left(s_{i}(t)\right)\right]
$$

The comparison simulation results of the three control strategies are shown in Figures 3(d)-4(a). Figure 3(d) shows the frequency deviation of each area under different control strategies; Figure 4(a) shows the power deviation on the tie line between each area and the other areas under different control strategies.

The simulation results show that, under the influence of the same load disturbances, the PI-type load frequency controller cannot quickly respond to the change of the load disturbances, resulting in a large system frequency deviation and tie-line power deviation, and it is difficult to maintain the stability of the grid. The traditional sliding mode controller has a better control effect by virtue of its own sliding mode variable structure, but its control performance is slightly lacking due to the difficulty of setting the appropriate disturbance boundary value. The interval disturbance reconstruction based sliding mode load frequency control strategy can effectively and quickly respond to load changes and maintain the system frequency deviation within a small range of changes.

5.2. Case 2. In actual engineering, the power grid is not only affected by load disturbances in different areas but also faces the problem of sensor fault due to the different characteristics of the sensor itself and the interference of the operating environment. Therefore, in this case, in addition to the different load disturbances in the three areas in Case 1, the problem of sensor fault in the system is also considered, and simulation experiments are carried out. For the sake of simplicity, suppose the form of sensor fault signal is as follows and sensor fault occurs after $50 \mathrm{~s}$ : 


$$
\begin{aligned}
& f_{s 1}(t)=\left[\begin{array}{llll}
f_{s 1,1}(t) & 0 & 0 & 0
\end{array}\right]^{T}, \\
& f_{s 2}(t)=\left[\begin{array}{llll}
f_{s 2,1}(t) & 0 & 0 & 0
\end{array}\right]^{T}, \\
& f_{s 3}(t)=\left[\begin{array}{llll}
f_{s 3,1}(t) & 0 & 0 & 0
\end{array}\right]^{T},
\end{aligned}
$$

where $f_{s 1,1}(t), f_{s 2,1}(t)$, and $f_{s 3,1}(t)$ are shown in Figure 4(d).

Interval residual errors based on interval observers have considered system disturbances, so there will be no false alarms due to external disturbances, which can be used for fault diagnosis. Figures 4(b) and 4(c) show the interval residual errors of $\Delta f_{i}(t)$ and $\Delta P_{t i e, i}(t)$, respectively. When the system is operating normally, the interval residual errors satisfy $0 \in\left[r_{i s}^{-}(t), r_{i s}^{+}(t)\right]$, but when faults occur, there are $0 \notin\left[r^{-}\left[\Delta f_{i}\right], r^{+}\left[\Delta f_{i}\right]\right]$ and $0 \in\left[r^{-}\left[\Delta P_{t i e, i}\right], r^{+}\left[\Delta P_{t i e, i}\right]\right]$, from which the fault locations can be diagnosed.

The sensor fault signals will be estimated by the augmented observer online, once the fault is detected. The estimated value of the sensor faults is shown in Figure 4(d), from which we can find that the augmented observer can well track and estimate the sensor fault signals quickly.

Figures 5(a) and 5(b) show $\Delta f_{i}(t)$ and $\Delta P_{t i e, i}(t)$ response, respectively, in each area after sensor faults occur. It can be seen from the simulation experiment that, in the case of the same fault, the controller without considering the fault compensation is difficult to avoid the interference of sensor fault, so that the system frequency and the tie-line power have a large deviation, and it is difficult to maintain the stability of the grid. However, the controller based on fault compensation can well reduce the influence of faults on the system, so that the system frequency and the deviation of tieline power can be kept within a small range and the stability of the grid can be maintained.

\section{Conclusion}

A new LFC scheme based on load disturbance reconstruction is proposed for the multiarea power grid, and the corresponding FTC strategy is also developed in this paper. The conclusions of this paper are as follows:

(1) The disturbance is reconstructed based on interval estimation, which solves the problem of decoupling and reconstruction of load disturbance.

(2) An integral sliding mode controller based on disturbance reconstruction is designed to ensure that the frequency deviation of the system is small. The simulation results show that the proposed controller can respond to the load changes effectively and quickly and the system frequency deviation can be kept in a small range.

(3) Sensor faults are diagnosed by interval residual. An augmented observer is designed to estimate sensor faults. The results show that the influence of sensor faults on system performance is greatly eliminated by using sensor fault estimation to compensate the system, and the system runs reliably and stably.
In the future research work, we will also study how to reduce the influence of malicious attacks on the power grid and ensure the stable operation of the power grid.

\section{Data Availability}

The data used to support the findings of this study cannot be made freely available. Access to the data is restricted.

\section{Conflicts of Interest}

The authors declare that they have no conflicts of interest.

\section{Acknowledgments}

This work was partially supported by the National Natural Science Foundation of China (61973140) and Beijing Natural Science Foundation (21JC0026).

\section{References}

[1] A. Ameli, A. Hooshyar, E. F. El-Saadany, and A. M. Youssef, "Attack detection and identification for automatic generation control systems," IEEE Transactions on Power Systems, vol. 33, no. 5, pp. 4760-4774, 2018.

[2] Y. Arya, "ICA assisted (FTIDN)-D-lambda controller for AGC performance enrichment of interconnected reheat thermal power systems," Journal of Ambient Intelligence and Humanized Computing, vol. 15, no. 3, 2021.

[3] C. Li, Y. Wu, Y. Sun et al., "Continuous under-frequency load shedding scheme for power system Adaptive frequency control," IEEE Transactions on Power Systems, vol. 35, no. 2, pp. 950-961, 2020.

[4] C. Wang, Y. Mi, Y. Fu, and P. Wang, "Frequency control of an isolated micro-grid using double sliding mode controllers and disturbance observer," IEEE Transactions on Smart Grid, vol. 9, no. 2, pp. 923-930, 2018.

[5] D. Xu, J. Liu, X.-G. Yan, and W. Yan, “A novel adaptive neural network constrained control for a multi-area interconnected power system with hybrid energy storage," IEEE Transactions on Industrial Electronics, vol. 65, no. 8, pp. 6625-6634, 2018.

[6] M. Sharma, S. Dhundhara, Y. Arya, and S. Prakash, "Frequency excursion mitigation strategy using a novel COA optimised fuzzy controller in wind integrated power systems," IET Renewable Power Generation, vol. 14, no. 19, pp. 40714085, 2020.

[7] F. Zhu, X. Zhou, Y. Zhang, D. Xu, and J. Fu, "A load frequency control strategy based on disturbance reconstruction for multi-area interconnected power system with hybrid energy storage system," Energy Reports, vol. 7, pp. 8849-8857, 2021.

[8] M. Sharma, S. Dhundhara, Y. Arya, and S. Prakash, "Frequency stabilization in deregulated energy system using coordinated operation of fuzzy controller and redox flow battery," International Journal of Energy Research, vol. 45, no. 5, pp. 7457-7475, 2021.

[9] M. Elsisi, M. Soliman, M. A. S. Aboelela, and W. Mansour, "Improving the grid frequency by optimal design of model predictive control with energy storage devices," Optimal Control Applications and Methods, vol. 39, no. 1, pp. 263-280, 2018.

[10] M. Q. Tran, M. Elsisi, K. Mahmoud, M. K. Liu, M. Lehtonen, and M. M. F. Darwish, "Experimental setup for online fault diagnosis of induction machines via promising IoT and 
machine learning: towards industry 4.0 empowerment," IEEE access, vol. 9, pp. 115429-115441, 2021.

[11] M. Elsisi, M. Q. Tran, K. Mahmoud, D. E. A. Mansour, M. Lehtonen, and M. M. F. Darwish, "Towards secured online monitoring for digitalized GIS against cyber-attacks based on IoT and machine learning," IEEE Access, vol. 9, 2021.

[12] S. Yin, H. Gao, J. Qiu, and O. Kaynak, "Descriptor reducedorder sliding mode observers design for switched systems with sensor and actuator faults," Automatica, vol. 76, pp. 282-292, 2017.

[13] M. Liu and P. Shi, "Sensor fault estimation and tolerant control for Itô stochastic systems with a descriptor sliding mode approach," Automatica, vol. 49, no. 5, pp. 1242-1250, 2013.

[14] K. Wang, Y. Shao, L. Xie, J. Wu, and S. Guo, "Adaptive and fault-tolerant data processing in healthcare IoT based on fog computing," IEEE Transactions on Network Science and Engineering, vol. 7, no. 1, pp. 263-273, 2020.

[15] X. Su, X. Liu, and Y.-D. Song, "fault-tolerant control of multiarea power systems via a sliding-mode observer technique," IEEE, vol. 23, no. 1, pp. 38-47, 2018.

[16] J. Wu, Z. Chen, Y. Zhang, Y. Xia, and X. Chen, "Sequential recovery of complex networks suffering from cascading failure blackouts," IEEE Transactions on Network Science and Engineering, vol. 7, no. 4, pp. 2997-3007, 2020.

[17] N. Jaleeli, L. S. VanSlyck, D. N. Ewart, L. H. Fink, and A. G. Hoffmann, "Understanding automatic generation control," IEEE Transactions on Power Systems, vol. 7, no. 3, pp. 1106-1122, 1992.

[18] H. A. Yousef, K. Al-Kharusi, M. H. Albadi, and N. Hosseinzadeh, "Load frequency control of a multi-area power system: an adaptive fuzzy logic approach," IEEE Transactions on Power Systems, vol. 29, no. 4, pp. 1822-1830, 2014.

[19] S. Kayalvizhi and D. M. Vinod Kumar, "Load frequency control of an isolated micro grid using fuzzy adaptive model predictive control," IEEE Access, vol. 5, pp. 16241-16251, 2017.

[20] S. Trip and C. De Persis, "Distributed optimal load frequency control with non-passive dynamics," IEEE Transactions on Control of Network Systems, vol. 5, no. 3, pp. 1232-1244, 2018.

[21] C. Chen, M. Cui, X. Fang, B. Ren, and Y. Chen, "Load altering attack-tolerant defense strategy for load frequency control system," Applied Energy, vol. 280, Article ID 116015, 2020.

[22] A. M. Ersdal, L. Imsland, K. Uhlen, D. Fabozzi, and N. F. Thornhill, "Model predictive load-frequency control taking into account imbalance uncertainty," Control Engineering Practice, vol. 53, pp. 139-150, 2016.

[23] A. M. Ersdal, L. Imsland, and K. Uhlen, "Model predictive load-frequency control," IEEE Transactions on Power Systems, vol. 31, no. 1, pp. 777-785, 2016.

[24] H. Bevrani, Robust Power System Frequency Control, Springer, New York, NY, USA, 2009.

[25] P. Ojaghi and M. Rahmani, "LMI-based robust predictive load frequency control for power systems with communication delays," IEEE Transactions on Power Systems, vol. 32, no. 5, pp. 4091-4100, 2017.

[26] X. Shang-Guan, Y. He, C. Zhang, L. Jiang, J. W. Spencer, and $\mathrm{M}$. Wu, "Sampled-data based discrete and fast load frequency control for power systems with wind power," Applied Energy, vol. 259, Article ID 114202, 2020.

[27] Y. Mi, Y. Fu, D. D. Li, C. S. Wang, P. C. Loh, and P. Wang, "The sliding mode load frequency control for hybrid power system based on disturbance observer," International Journal of Electrical Power \& Energy Systems, vol. 74, pp. 446-452, 2016.
[28] Z. Wang, C.-C. Lim, and Y. Shen, "Interval observer design for uncertain discrete-time linear systems," Systems \& Control Letters, vol. 116, pp. 41-46, 2018.

[29] J. Li, Z. Wang, W. Zhang, T. Raïssi, and Y. Shen, "Interval observer design for continuous-time linear parameter-varying systems," Systems \& Control Letters, vol. 134, Article ID 104541, 2019.

[30] S. Guo, B. Jiang, F. Zhu, and Z. Wang, "Luenberger-like interval observer design for discrete-time descriptor linear system," Systems \& Control Letters, vol. 126, pp. 21-27, 2019.

[31] F. Xu, J. Tan, X. Wang, and B. Liang, "Conservatism comparison of set-based robust fault detection methods: settheoretic UIO and interval observer cases," Automatica, vol. 105, pp. 307-313, 2019.

[32] M. Liu, P. Shi, L. Zhang, and X. Zhao, "fault-tolerant control for nonlinear markovian jump systems via proportional and derivative sliding mode observer technique," IEEE Transactions on Circuits and Systems I: Regular Papers, vol. 58, no. 11, pp. 2755-2764, 2011.

[33] F. Boem, A. J. Gallo, D. M. Raimondo, and T. Parisini, "distributed fault-tolerant control of large-scale systems: an active fault diagnosis approach," IEEE Transactions on Control of Network Systems, vol. 7, no. 1, pp. 288-301, 2020.

[34] A. M. H. Teixeira, J. Araújo, H. Sandberg, and K. H. Johansson, "Distributed sensor and actuator reconfiguration for fault-tolerant networked control systems," IEEE Transactions on Control of Network Systems, vol. 5, no. 4, pp. 1517-1528, 2018.

[35] X. Su, P. Shi, L. Wu, and Y.-D. Song, "fault detection filtering for nonlinear switched stochastic systems," IEEE Transactions on Automatic Control, vol. 61, no. 5, pp. 1310-1315, 2016.

[36] C. Ke, C. Li, and Q. Zhang, "Distributed adaptive fault-tolerant consensus of nonlinear multi-agent systems via stateconstraint impulsive protocols with time-delay," IEEE Transactions on Network Science and Engineering, vol. 7, no. 4, pp. 3112-3121, 2020.

[37] X. G Yan and C. Edwards, "Adaptive sliding-mode-observerbased fault reconstruction for nonlinear systems with parametric uncertainties," IEEE Transactions on Industrial Electronics, vol. 55, no. 11, pp. 4029-4036, 2008.

[38] A. J. Wood and B. F. Wollenberg, Power Generation, Operation, and Control, Wiley, New York, NY, USA, 2012.

[39] L. Farina and S. Rinaldi, Positive Linear Systems: Theory and Applications, Wiley, New York, NY, USA, 2000.

[40] P. Gahinet and P. Apkarian, "A linear matrix inequality approach toHo control," International Journal of Robust and Nonlinear Control, vol. 4, no. 4, pp. 421-448, 1994. 